\title{
THE ROTATION OF THE AP STARS FROM THE POINT OF VIEW \\ OF THE RIGID ROTATOR MODEL
}

\author{
GEORGE W. PRESTON \\ Hale Observatories, Carnegie Institution of Washington, \\ California Institute of Technology, Calif., U.S.A.
}

\begin{abstract}
Deutsch's period vs. line-width relation for the periodic Ap stars is re-examined from the point of view of the rigid rotator with the aid of recently determined values of $v \sin i$ for these objects. The agreement between computed rotational velocities and observed values of $v \sin i$ is satisfactory if the radii of the Ap stars are twice those of zero-age main sequence stars of the same color. The computed rotational velocities also agree with the mean rotational velocities of all $\mathrm{Ap}$ stars if suitable allowances are made for observational limitations. However, a difficulty arises in connection with the number of 'long-period' stars that have been discovered. Their small computed rotational velocities and their frequency are such that they cannot be regarded as part of any rotational distribution function that describes normal stars or even most Ap stars. It is concluded that if the periods of these objects are rotational periods, then a powerful rotational deceleration mechanism must be operative in at least some Ap stars, primarily those of the $\mathrm{SrCrEu}$ group.
\end{abstract}

\section{Introduction}

During the past few years the number of known Ap stars with well-determined periods of light, spectrum, or magnetic variations has been increased sufficiently to make feasible a re-examination of Deutsch's (1956) period vs. line-width relation. Such a study is of interest from two points of view: (1) to determine the radii of Ap stars that are required to satisfy the observations on the assumption that these objects are rigid rotators, (Stibbs, 1950; Deutsch, 1954) and (2) to compare the rotational velocities calculated by means of these radii with the rotations of all Ap stars as inferred from their mean projected rotational velocities. The latter comparison provides an important consistency check on the rotator model.

Arguments for or against the rigid rotator have been advanced in several summary discussions (Deutsch, 1958; Babcock 1960; Ledoux and Renson, 1966) by authors of dissimilar points of view. However, it is generally agreed that the rotator model explains the period vs. line-width relation in a natural way, predicts the reversals of magnetic polarity that are commonly observed, and explains the order of magnitude, sign convention, and phases of the crossover effect (Babcock, 195I, 1956). Objections to the hypothesis that these stars possess 'apparent abundance patches' have recently been countered by strong evidence that the magnetic field variations of different elements are not alike in at least two stars, HD 188041 (Preston, 1967a; Wolff, 1969) and $\beta \mathrm{CrB}$ (Preston and Sturch, 1967). This result requires a spatial separation of the elements involved. There remain two long-standing objections to the rigid rotator model. The first is that it does not predict irregular magnetic activity or irregular spectrum variability. The first counter to this objection appeared when Steinitz (1964) discovered the period of $\beta \mathrm{CrB}$, the prototype of irregular magnetic variables with reversing polarity. Since then magnetic periods for HD 10783 (Steinitz, 1964), 
HD 133029 (Renson reported by Herbig, 1967), and HD 215441 (Preston, 1969a) have been reported. Even more recently, the periodic spectrum variability of 21 Per (heretofore regarded as an irregular spectrum variable) and the periodic magnetic and spectrum variability of $17 \mathrm{Com} A$ and $78 \mathrm{Vir}$ (the classic example of an apparently irregular magnetic star) have been established (Preston 1969b, c; Preston et al., 1969). Therefore, at this juncture it is my opinion that the burden of proof lies with those who believe that irregular magnetic or spectrum variations exist.

The final objection to the rigid rotator concerns the fact that the photometric and magnetic periods of any particular Ap star are always identical. Up to the present time there has been no explanation of why surface brightness should depend on magnetic polarity. However, a reason may be forthcoming in view of recent observations of the mean surface fields of $\beta \mathrm{CrB}$ (Wolff and Wolff, 1970) and HD 126515 (Preston, 1970f). The mean surface field is the absolute value of the total surface field averaged over the visible hemisphere of a star; it can be derived accurately for a number of stars that possess fields sufficiently large to produce splitting of certain favorable Zeeman doublet patterns (Preston 1969d, e). For both $\beta$ CrB and HD 126515 this quantity also varies with a single wave in the period of the effective field, and visual inspection of spectrograms indicates that this is the case for $53 \mathrm{Cam}$ as well. In the case of HD 126515 the mean surface field varies between 10 and 17 kilogauss while the effective field varies between +2 and -2 kilogauss. This behavior can be explained qualitatively by a decentered field distribution, i.e. the two magnetic hemispheres are dissimilar with respect to their total magnetic field strengths. The disparity amounts to a factor of $\simeq 2$ in $H$ and hence to a factor of $\simeq 4$ in the mean magnetic energy density, $H^{2} / 8 \pi$ of opposing hemispheres. Thus it is conceivable, though not yet demonstrated, that this asymmetry in the field distribution is related to the aspectdependant luminosity that is required by the rigid-rotator model.

In summary then, it does not appear that the rigid rotator can be discounted by virtue of any of the objections discussed above. We, therefore, proceed to the present investigation.

\section{The Observations}

During the course of a survey of rotational velocities of the Ap stars, values of $v \sin i$ were obtained for all of the well-studied periodic Ap stars known to the writer. These are listed in Table I. With one or two exceptions, for which a prima facie case for variability could be made from one set of observational data, the list is restricted to stars whose variations have been confirmed by independent sets of observations or for which a single period has been derived for two different kinds of variations (light, spectrum, magnetic field). The values of $v \sin i$ were derived from a comparison of measured line-widths on coudé spectrograms with those of a set of rotational standard stars. The projected rotational velocities of these standard stars were derived by a procedure to be described in detail elsewhere. The linear dispersions employed in the survey ranged from 1.3 to $10 \AA \mathrm{mm}^{-1}$ and for the periodic stars were generally matched to the stars so that the instrumental profiles did not seriously distort the 


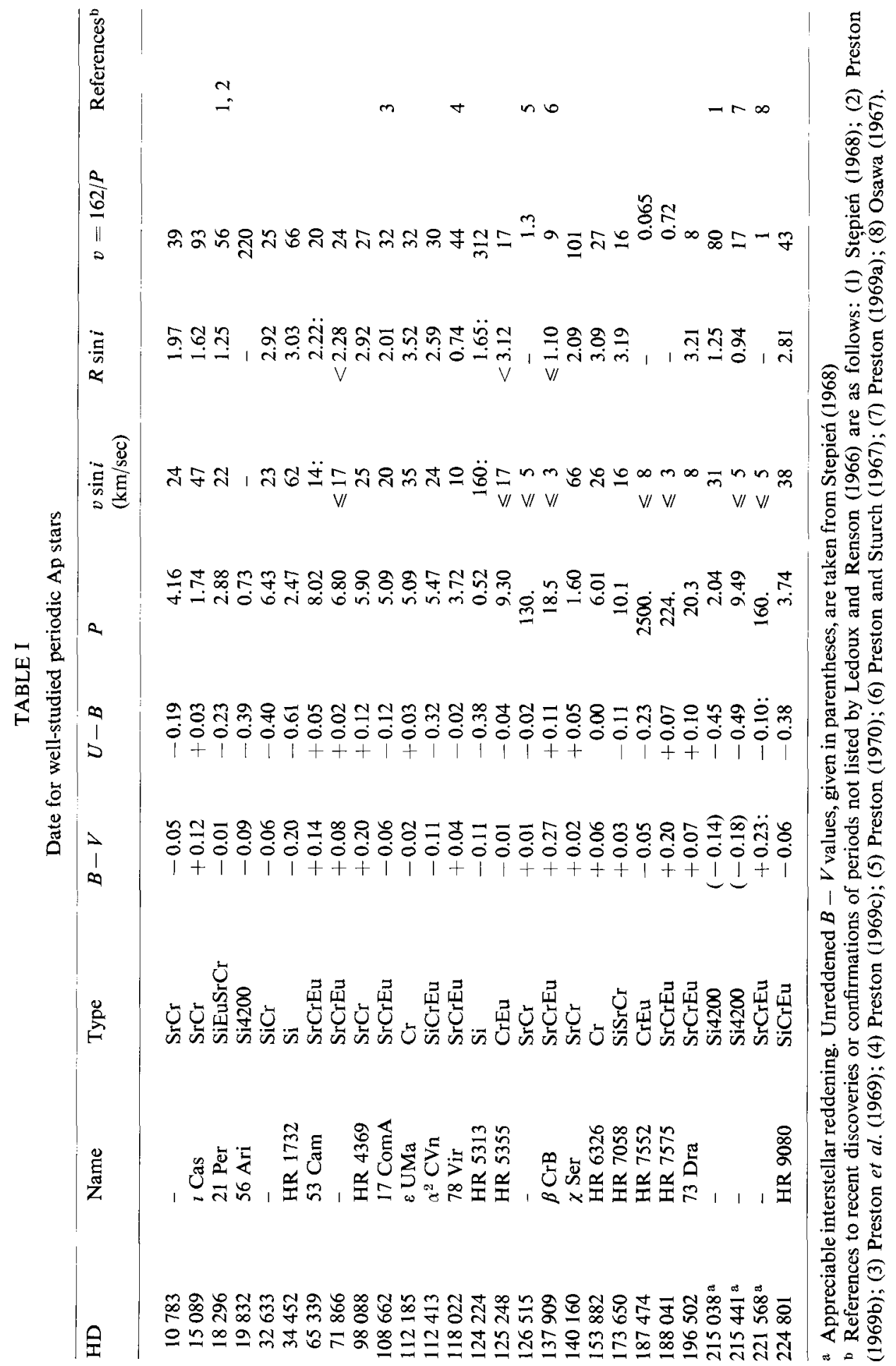


stellar profiles except in the cases of HD 126515, $\beta$ CrB, HD 188041, HD 215441, and HD 221568; for these objects 1 could only establish approximate upper limits to $v \sin i$, as indicated in column 7 of Table I. The values of $v \sin i$ for $\beta \mathrm{CrB}$ and HD 188041 are derived from the width of the null line Fe I $\lambda 4065.40$ (see Preston, 1967b); for HD 126515 and HD 215441, the widths of resolved Zeeman line components were used. The Zeeman effect is known to produce measurable line broadening in some magnetic stars (Preston 1967a, b; 1969d) and it seriously affects the line widths of $53 \mathrm{Cam}, \mathrm{HD} 71866$, HD 125248, and HD 187474. The upper limits on $v \sin i$ for these objects were obtained from the widths of polarized line components as observed with a Babcock-type differential circular analyzer.

The stars in Table II were omitted from the discussion because their reported periods lack confirmation (if no notes are appended) or are doubtful or in dispute as indicated by the Notes in column 8 . Hopefully, many of these reported periods will be confirmed by observers in the near future.

\section{Radius Estimates}

For a rigid rotator the equatorial rotational velocity $v$, the period $P$, and the stellar radius $R$ are related by $2 \pi R=P v$, or

$$
R=P v / 50.6
$$

if $R, v$, and $P$ are expressed in solar radii, $\mathrm{km} \mathrm{sec}^{-1}$, and days, respectively. The stars are viewed at unknown inclination $i$ so that Equation (1) takes the form

$$
R \sin i=(P / 50.6)(v \sin i)
$$

in a discussion of projected rotational velocities. If the $i$ values of the periodic Ap stars are randomly distributed, then for a set of stars with constant $R$ we have $R=(4 / \pi)\langle R \sin i\rangle$ or

$$
R=0.025\langle P v \sin i\rangle .
$$

If we apply Equation (3) to the data in Table I we obtain $R=2.9 R_{\odot}$. However, inspection of Figure 1 indicates that this value is not satisfactory because too many stars with well-determined periods and values of $v \sin i$ exceed it. This result could be anticipated from the observed frequency distribution of $R \sin i$ which is not proportional to $\tan i$, as it should be. This result can arise if $R \not \equiv$ constant or if there is an observational bias in favor of short periods at a given $v \sin i$ and vice versa. I suspect that both biases exist because photometric and spectroscopic observing programs frequently have been designed to study short - rather than long - period variations, and there is an obvious bias in favor of detection of spectrum variations in sharp-lined stars. Therefore, I have used an upper-envelope method to estimate the value $R=3.2$ $R_{\odot}$; a zero-age main sequence curve scaled by a factor of 2 , as indicated in Figure 1 would serve as well or better. In either case the rigid rotator requires that a major fraction and perhaps all of the Ap stars have radii that exceed those of zero-age main sequence stars by a factor of $\simeq 2$. This places them $\simeq 1.5$ magnitudes above the zero- 


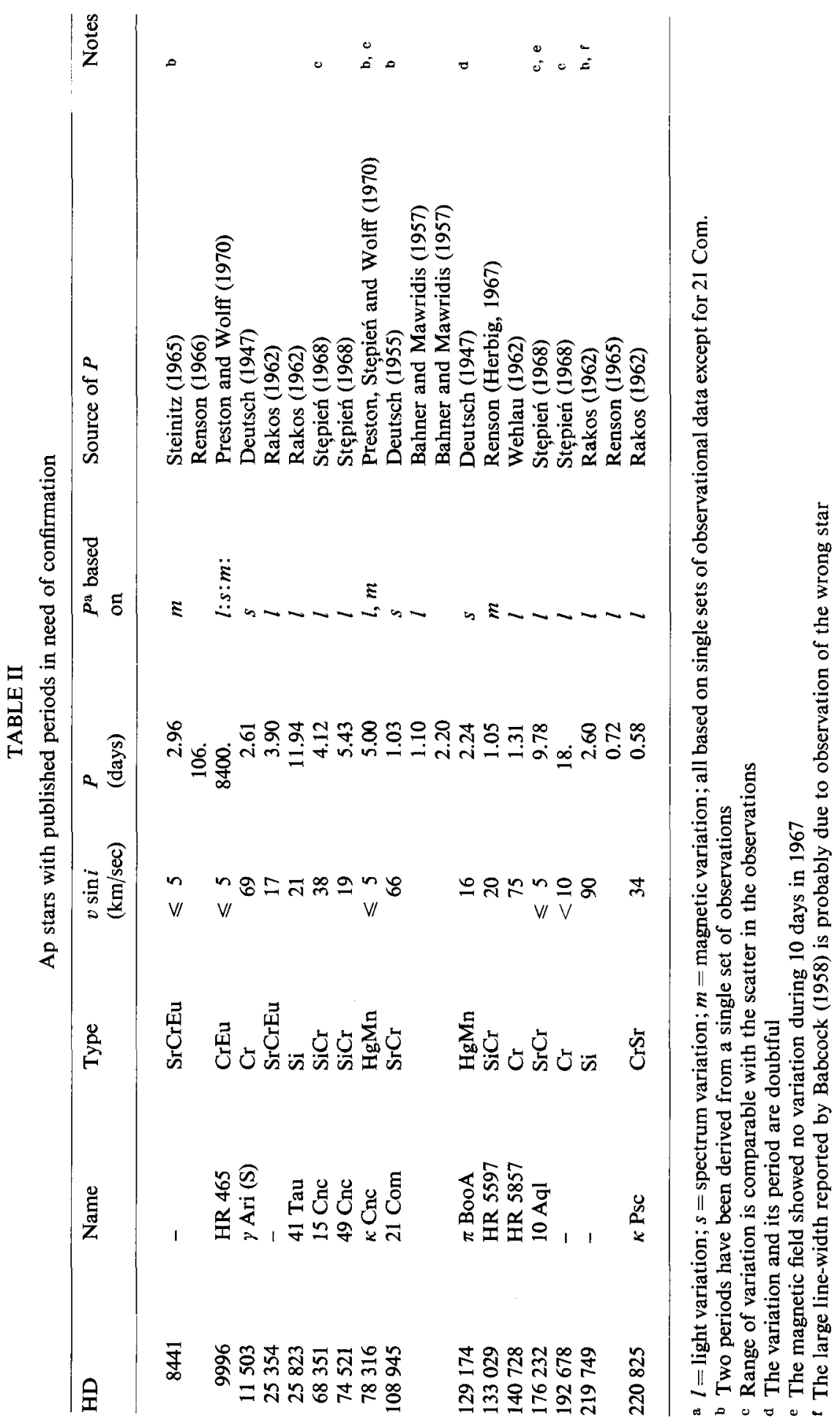


age main sequence, a result that is in reasonable agreement with the meager data available (see, for example Kraft, 1967, and Wolff, 1967).

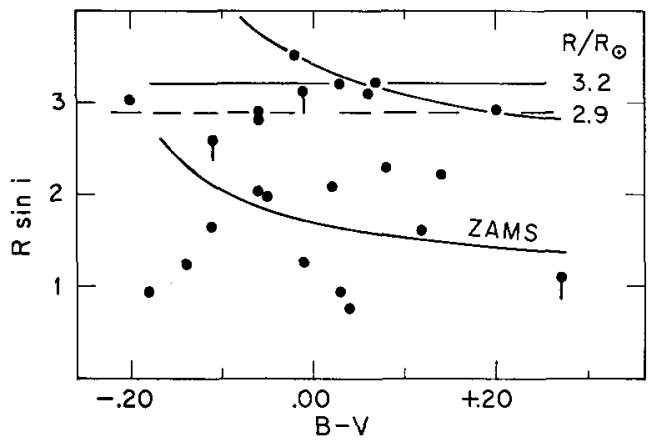

Fig. 1. A plot of $R \sin i$ in units of solar radii vs. $B-V$ for the periodic Ap stars listed in Table I. The radii of zero-age main sequence stars calculated from the data of Morton and Adams (1968) is indicated by ZAMS. The average and upper envelope estimates of $R / R_{\odot}=2.9$ and 3.2 are indicated by dashed and solid horizontal lines. A ZAMS curve, scaled by a factor of 2 is also shown. Vertical bars below three points denote upper limits on $R \sin i$.

\section{The Mean Rotational Velocities of the Periodic Ap Stars}

In Table III the rotational velocities of the periodic Ap stars are compared with those obtained from the survey of all Ap stars mentioned above. The survey results are preliminary ones based on about $90 \%$ of the total sample. Because the mean rotational velocities of various Ap subgroups are not alike, I have presented the results separately for the two main groups, the Si stars and the SrCrEu stars. Any star with enhanced $\mathrm{Si}$ II lines is treated as a $\mathrm{Si}$ star regardless of its other properties. Thus, in this paper $\alpha^{2} \mathrm{CVn}$ and 21 Per are regarded as Si stars in spite of the fact that their spectra show other more striking peculiarities. The types are taken primarily from Osawa (1965). Some of them will be revised in the final discussion of the survey data but it is not likely that such revisions or the addition of $\simeq 30$ more stars will affect the results qualitatively. The quantities $\langle v \sin i\rangle$ and $\langle v\rangle=(4 / \pi)\langle v \sin i\rangle$ for the survey stars are

TABLE III

Rotational velocity data for Ap stars

\begin{tabular}{llrc} 
& & Si & SrCrEu a \\
\hline$\langle v \sin i\rangle$ & All Ap & 53 & $<29$ \\
& Periodic Ap & 42 & $<19$ \\
$\langle v\rangle$ & All Ap & 67 & $<37$ \\
$\langle v(P)\rangle$ & Periodic Ap & 86 & 28 \\
No. of & All Ap & 100 & 88 \\
stars & Periodic Ap & 10 & 17
\end{tabular}

a The inequalities result from the fact that the line-widths in many members of the $\mathrm{SrCrEu}$ group are smaller than the instrumental width. Upper limits on $v \sin i$ for such stars were included in the averages. 
based on substantial samples as indicated at the bottom of Table III. The mean rotational velocities of the periodic stars were obtained from the data in Table I by means of Equation (1) with $R=3.2 R_{\odot}$. The agreement between the results for all Ap stars and for the periodic Ap stars is satisfactory. In this assessment allowance must be made for the following: (1) the small sample of periodic stars, (2) the inclusion of 56 Ari in the $\langle v(P)\rangle$ calculation and its exclusion in the $\langle v \sin i\rangle$ calculation for the periodic Si stars, and (3) the fact that the periodic SrCrEu stars have been systematically observed at higher dispersion than those in the survey. This results in smaller upper limits on $v \sin i$ for a number of the periodic stars (no account has been taken of inequality signs in the computation of average values). In summary, then, it is possible to choose radii for the periodic stars so that their rotational velocities, as calculated for the rigid-rotator model, are in no way anomalous with respect to the rotations of all Ap stars.

\section{The Long-Period Ap Stars}

There remains one disquieting feature of the observational data from the point of view of the rigid rotator model - the number of long-period Ap stars and the rotational velocities inferred from their periods. There are now six known or suspected Ap stars with periods greater than 100 days as indicated in Table IV. The

\begin{tabular}{rccl}
\multicolumn{3}{c}{ TABLE IV } \\
HD & $\begin{array}{c}\text { mag. } \\
\text { m (days) }\end{array}$ & $v(P)$ \\
8441 & 6.6 & $106(?)$ & $1.5(?) \mathrm{km} / \mathrm{sec}$ \\
126515 & 7.0 & 130 & 1.2 \\
221568 & 8.0 & 160 & 1.0 \\
188041 & 5.6 & 224 & 0.72 \\
187474 & 5.4 & $2500:=6.7$ years & 0.065 \\
9996 & 6.3 & long: 23 years $(?)$ & $0.019(?)$
\end{tabular}

suggested magnetic period for HD 8441 is also the spectroscopic binary period of that star according to Renson (1966); the period of HD 126515 is of recent origin (Preston, 1970) and definite; and those for HD 221 568, HD 188041, and HD 187474 all seem secure. The extraordinary period of HD 9996 given above must be regarded as tentative because of an inadequate number and distribution of observations, but the variation is certainly slow as indicated not only by a rare earth spectrum variation, but by fragmentary magnetic and photometric data as well; this star is also a spectroscopic binary. None of these stars are extraordinary among the Ap stars from the spectroscopic point of view. Their lines are very sharp, but this is a common feature of the $\mathrm{SrCrEu}$ group to which they all belong. Five of the six star are spectrum variables and for at least three of them the spectrum and magnetic variations proceed in phase as is generally the case for magnetic stars.

Astronomers will generally view reports of rotational velocities less than $0.1 . \mathrm{km}$ 
$\sec ^{-1}$ with some reluctance and the following illustrative calculations indicate why. The Maxwellian distribution gives a fair representation of rotational velocities for normal upper main sequence stars (see, for example Babcock, 1960, and Deutsch, 1967). This distribution may be written

$$
f(x)=\frac{4}{\sqrt{ } \pi} x^{2} e^{-x^{2}},
$$

where $x=v / v_{\mathrm{m}}$ is the velocity in units of the modal velocity of the distribution. The fraction of a Maxwellian population that has $x$ in $0 \leqslant x \leqslant X$ is

$$
F(X)=\int_{0}^{x} f(x) \mathrm{d} x \approx 0.75 X^{3} \quad \text { if } \quad X \ll 1 .
$$

There are $\simeq 10^{3}$ B5-A3 stars brighter than $m_{\mathrm{v}}=6$ and thus there are certainly $<10^{4}$ such stars brighter than $m_{\mathrm{v}}=7$. For these objects $v_{\mathrm{m}} \simeq 150 \mathrm{~km} \mathrm{sec}^{-1}$. There are $\simeq 4 \pm 1$ known long-period Ap stars with $v \leqslant 1.5 \mathrm{~km} \mathrm{sec}^{-1}$ and $m_{\mathrm{v}} \leqslant 7$. The expected number for $X=1.5 / 150=0.01$ is $\simeq 10^{4} F(0.01) \simeq 10^{-2}$; there is a discrepancy of a factor of 400 . Suppose that the long-period stars are the slowest rotators of an Ap distribution with $v_{\mathrm{m}}=30 \mathrm{~km} \mathrm{sec}^{-1}$ If the Ap stars comprise $10 \%$ of the normal stars, then the expected number with $v<1.5 \mathrm{~km} \mathrm{sec}^{-1}$ is $\simeq 10^{3} F(0.05) \simeq 10^{-1}$; there is a discrepancy of a factor of 40 . The actual discrepancies must be much larger than those calculated above because the list of long-period stars can hardly be expected to be complete. Thus it does not seem possible to regard these objects as members of any known velocity distribution. If their periods are rotational periods, then a powerful deceleration mechanism must be operative in a substantial fraction of the Ap stars, primarily those of the $\mathrm{SrCrEu}$ group. If their periods are not rotational periods, then, by virtue of the similarity of their variations to those of other Ap stars of shorter period, I believe it will be necessary to abandon the rigid-rotator model.

\section{Acknowledgement}

The writer wishes to acknowledge helpful discussions with Dr. A. J. Deutsch during the preparation of this report.

\section{References}

Babcock, H. W.: 1951, Astrophys. J. 114, 1.

Babcock, H. W.: 1956, Astrophys. J. 124, 489.

Babcock, H. W.: 1958, Astrophys. J. Suppl. 3, No. 30.

Babcock, H. W.: 1960, in Stars and Stellar Systems 6, p. 282.

Bahner, K. and Mawridis, L.: 1957, Z. Astrophys. 41, 254.

Deutsch, A. J.: 1947, Astrophys. J. 105, 283.

Deutsch, A. J.: 1954, Trans. IAU 8, 801.

Deutsch, A. J.: 1955, Publ. Astron. Soc. Pacific 67, 342.

Deutsch, A. J.: 1956, Publ. Astron. Soc. Pacific 68, 92. 
Deutsch, A. J.: 1958, Handbuch der Physik 51, 689.

Deutsch, A J : 1967, in The Magnetic and Related Stars (ed. by R. Cameron), Mono Book Corp., Baltimore, p. 181.

Herbig, G. H.: 1967, Trans. IAU 13A, 523.

Kraft, R. P.: 1967, in The Magnetic and Related Stars (ed. by R. Cameron), Mono Book Corp. Baltimore, p. 303.

Ledoux, P. and Renson, P.: 1966, Ann. Rev. Astron. Astrophys. 4, 293.

Morton, D. C. and Adams, T. F.: 1968, Astrophys. J. 151, 611.

Osawa, K.: 1965, Ann. Tokyo Astron. Obs., Ser. 2, 9, 123.

Osawa, K.: 1967, in The Magnetic and Related Stars (ed. by R. Cameron), Mono Book Corp., Baltimore, p. 363.

Preston, G. W.: 1967a, in The Magnetic and Related Stars (ed. by R. Cameron), Mono Book Corp., Baltimore, p. 3.

Preston, G. W.: 1967b, Astrophys. J. 147, 804.

Preston, G. W.: 1969a, Astrophys. J. 156, 967.

Preston, G. W.: 1969b, Astrophys. J. 158, 243.

Preston, G. W.: 1969c, Astrophys. J. 158, 251.

Preston, G. W.: 1969d, Astrophys. J., 157, 247.

Preston, G. W.: 1969e, Astrophys. J. 158, 1081.

Preston, G. W.: 1970, Astrophys. J. 160, in press.

Preston, G. W. and Sturch, C.: 1967, in The Magnetic and Related Stars (ed. by R. Cameron), Mono Book Corp., Baltimore, p. 363.

Preston, G. W. and Wolff, S. C.: 1970, Astrophys. J. 160, in press.

Preston, G. W., Stẹpień, K., and Wolff, S. C.: 1969, Astrophys. J. 156, 653.

Rakos, K.: 1962, Lowell Obs. Bull. 5, 227.

Renson, P.: 1965, Bull. Soc. Roy. Sci. Liège, 34, No. 5-6, 302.

Renson, P.: 1966, Bull. Soc. Roy. Sci. Liège, 35, No. 3-4, 244.

Steinitz, R.: 1964, Bull. Astron Inst. Netherl. 17, 504.

Steinitz, R.: 1965, Bull. Astron. Inst. Netherl. 18, 125.

Stepień, K.: 1968, Astrophys. J. 154, 945.

Stibbs, D. W. N.: 1950, Monthly Notices Roy. Astron. Soc. 110, 395.

Wehlau, W.: 1962, Pub. Astron. Soc. Pacific, 74, 286.

Wolff, S. C.: 1967, Astrophys. J. Suppl. Ser. 15, 21.

Wolff, S. C.: 1969, Astrophys. J. 157, 253.

Wolff, S. C. and Wolff, R.: 1970, Astrophys. J., in press.

\section{Discussion}

Van den Heuvel: Is there any evidence that the last six stars you listed are considerably older than the other Ap stars? If a braking mechanism is going on, they must be the oldest Ap stars present.

Preston: These stars are all members of the $\mathrm{SrCrEu}$ group and hence are systematically cooler and presumably older than, say, the Si stars. However, I have no way of determining the relative ages of stars within the SrCrEu group. In any event, I think it would be premature to regard the long-period stars as the oldest ones until we understand the nature of the braking mechanism.

Van den Heuvel: What braking mechanism for the rotation of Ap stars would you like to propose?

Preston: For some time I have been looking for observational evidence of an interaction with the interstellar medium. During these meetings Dr. Ostriker has suggested to me one such interaction which involves the dissipation of rotationai energy by Alfvén waves generated at the boundary of a stellar magnetosphere.

Abt: Could you please explain more carefully how you obtain two different measures of the magnetic field?

Preston: The effective field is the mean value of the longitudinal component of the magnetic field averaged over the stellar disk. It is derived from the displacement between the centroids of unresolved Zeeman patterns that have been analyzed for left and right circular polarization. The positions of the centroids are determined by the relative intensities of the Zeeman components as viewed through a differential circular analyzer. If the stellar magnetic field is sufficiently strong, then certain favorable 
Zeeman patterns are resolved and the separation of the resolved $\sigma$ components provides a direct measure of the total field averaged over the stellar disk. A detailed discussion is given in the references cited in the text.

Bernacca: You derived mean true values such as $\langle v\rangle$ or $\langle R \sin i\rangle\langle\sin i\rangle$ by means of $\langle\sin i\rangle=$ $\pi / 4$. Now, according to my paper this averaging factor is not the most correct one that you can have for an observed sample of stars. If, for your Ap stars it can be assumed that the upper limit to the true velocities is the maximum observed apparent velocity, you get a different value for $\langle\sin i\rangle$. The question is the following: How sensitive are your conclusions to the above predicted mean values?

Preston: The two procedures gave radii that differed by about $10 \%$. However, as I indicated, observational selection effects and/or a dispersion in radii may be responsible for this difference.

Jaschek: Do magnetic fields repeat exactly cycle after cycle?

Preston: I do not know of any well-documented case of cyclic or random fluctuations in the shapes or ranges of periodic magnetic variations.

Jaschek: Do you have any mechanism for forming patches of $\mathrm{Cr}$ or $\mathrm{Eu}$ and holding them fixed on the surface?

Preston: I know of no mechanism for the formation of abundance patches. On the other hand a static magnetic field will act to preserve the identity of such patches once they have been formed. 